\title{
Simple $S L(n)$-modules with normal closures of maximal torus orbits
}

\author{
Karine Kuyumzhiyan
}

Received: 16 August 2008 / Accepted: 24 February 2009 / Published online: 16 March 2009

(C) Springer Science+Business Media, LLC 2009

\begin{abstract}
Let $T$ be the subgroup of diagonal matrices in the group $S L(n)$. The aim of this paper is to find all finite-dimensional simple rational $S L(n)$-modules $V$ with the following property: for each point $v \in V$ the closure $\overline{T v}$ of its $T$-orbit is a normal affine variety. Moreover, for any $S L(n)$-module without this property a $T$-orbit with non-normal closure is constructed. The proof is purely combinatorial: it deals with the set of weights of simple $S L(n)$-modules. The saturation property is checked for each subset in the set of weights.
\end{abstract}

Keywords Toric variety $\cdot$ Normality $\cdot$ Saturation

\section{Introduction}

Let $T$ be an algebraic torus defined over an algebraically closed field $\mathbb{k}$ of characteristic zero. Recall that an irreducible algebraic $T$-variety $X$ is called toric if $X$ is normal and $T$ acts on $X$ with an open orbit. This class of varieties plays an important role in algebraic geometry, topology and combinatorics due to its remarkable description in terms of convex geometry, see [6]. Assume that the torus $T$ acts on a variety $Y$. Then the closure $X=\overline{T y}$ of the $T$-orbit of a point $y \in Y$ is a natural candidate to be a toric variety. To verify it, one should check that $X$ is normal.

During last decades, normality of torus orbits' closures was an object of numerous investigations. For example, let $G$ be a semisimple algebraic group with a Borel subgroup $B$ and a maximal torus $T \subset B$. In [10], it was proved that the closure of a general $T$-orbit on the flag variety $G / B$ is normal. Later it was shown that the closure of a general $T$-orbit in $G / P$, where $P \subset G$ is a parabolic subgroup, is also normal,

K. Kuyumzhiyan $(\bowtie)$

Department of Higher Algebra, Faculty of Mechanics and Mathematics, Moscow State University, 119991 Moscow, Russia

e-mail: karina@mccme.ru 
see [5]. Examples of non-normal closures of non-general torus orbits can be found in [3].

Now let us consider a finite-dimensional rational $T$-module $V$. There exists an easy combinatorial criterion of normality of $\overline{T v}$ for a vector $v \in V$. Namely, let $v=$ $v_{\chi_{1}}+\cdots+v_{\chi_{m}}, v_{\chi_{i}} \neq 0$, be the weight decomposition of the vector $v$. Consider the corresponding set of $T$-weights $\chi_{1}, \ldots, \chi_{m}$. If we take $\chi_{1}, \ldots, \chi_{m}$ as elements of the character lattice $\mathfrak{X}(T)$, we can generate a semigroup $\mathbb{Z}_{+}\left(\chi_{1}, \ldots, \chi_{m}\right)$, a sublattice $\mathbb{Z}\left(\chi_{1}, \ldots, \chi_{m}\right)$, and a rational polyhedral cone $\mathbb{Q}_{+}\left(\chi_{1}, \ldots, \chi_{m}\right)$. The set $\chi_{1}, \ldots, \chi_{m}$ is called saturated if $\mathbb{Z}_{+}\left(\chi_{1}, \ldots, \chi_{m}\right)=\mathbb{Z}\left(\chi_{1}, \ldots, \chi_{m}\right) \cap \mathbb{Q}_{+}\left(\chi_{1}, \ldots, \chi_{m}\right)$. It is well known (see [9, page 5]) that the following two conditions are equivalent: the set $\left\{\chi_{1}, \ldots, \chi_{m}\right\}$ is saturated and the closure $\overline{T v}$ of the $T$-orbit $T v$ is normal. There is an analogous criterion for the $T$-action on the projectivisation $\mathbb{P}(V)$, see [3].

The saturation property occurs in many algebraic and geometric problems. In [17], it was proved that the set of incidence vectors of the bases of a realizable matroid is saturated. The geometric conclusion of this fact is that for any point $y$ in the affine cone over the classical Grassmannian $\operatorname{Gr}(k, n)$ the closure $\overline{T y}$ is normal.

Taken a finite graph $\Gamma$ with $n$ vertices, one can associate a finite collection $M(\Gamma)$ of vectors in the lattice $\mathbb{Z}^{n}$ with it:

$$
M(\Gamma)=\left\{\varepsilon_{i}+\varepsilon_{j}:(i j) \text { is an edge of } \Gamma\right\},
$$

where $\varepsilon_{1}, \varepsilon_{2}, \ldots, \varepsilon_{n}$ is the standard basis of $\mathbb{Z}^{n}$. The saturation property for this set is equivalent to the fact that for two arbitrary minimal odd cycles $C$ and $C^{\prime}$ in $\Gamma$, either $C$ and $C^{\prime}$ have a common vertex or there exists an edge of $\Gamma$ joining a vertex of $C$ with a vertex of $C^{\prime}$ (see [12] and [13]). Algebraically, the saturation property for $M(\Gamma)$ is equivalent to the integral closureness for the subalgebra $\mathcal{A}(\Gamma)$ of the polynomial algebra $\mathbb{k}\left[x_{1}, x_{2}, \ldots, x_{n}\right]$,

$$
\mathcal{A}(\Gamma)=\mathbb{k}\left[x_{i} x_{j}:(i j) \text { is an edge of } \Gamma\right],
$$

in its field of fractions $Q \mathcal{A}(\Gamma)$.

Some general results concerning quivers and the saturation property were obtained in [4]. It was shown that a finite, connected quiver $Q$ without oriented cycles is a Dynkin or Euclidean quiver if and only if all orbit semigroups of representations of $Q$ are saturated.

In the paper [11], the following problem is solved. Let $G$ be a semisimple algebraic group with a maximal torus $T$ and $V$ be its adjoint module. For which $G$ is the closure $\overline{T v}$ normal for all $v \in V$ ? The surprising fact is that for $G=S L(n)$ this is always the case (see [11, Thm.1], and also [15, Ex. 3.7], [14] and [2, Prop.2.1]). In [2], this combinatorial result is interpreted in terms of representations of quivers.

The aim of this paper is to classify all simple finite-dimensional rational $S L(n)$ modules $V$ such that for any $v \in V$ the closure $\overline{T v}$ is normal.

Main Theorem The representations below, together with their dual, form the list of all irreducible representations of $S L(n)$ where all maximal torus orbits' closures are normal:

(1) the tautological representation of $S L(n)$; 
(2) the adjoint representation of $S L(n)$;

(3) exceptional cases:

\begin{tabular}{|c|c|c|}
\hline Group & Highest weight & G-module \\
\hline$S L(2)$ & $3 \pi_{1}$ & $S^{3} \mathbb{k}^{2}$ \\
\hline$S L(2)$ & $4 \pi_{1}$ & $S^{4} \mathbb{k}^{2}$ \\
\hline$S L(3)$ & $2 \pi_{1}$ & $S^{2} \mathbb{k}^{3}$ \\
\hline$S L(4)$ & $\pi_{2}$ & $\Lambda^{2} \mathbb{k}^{4}$ \\
\hline$S L(5)$ & $\pi_{2}$ & $\Lambda^{2} \mathbb{k}^{5}$ \\
\hline$S L(6)$ & $\pi_{2}$ & $\Lambda^{2} \mathbb{k}^{6}$ \\
\hline$S L(6)$ & $\pi_{3}$ & $\Lambda^{3} \mathbb{k}^{6}$ \\
\hline
\end{tabular}

The paper is organized as follows. In Section 1 we give some algebraic definitions and reformulate the problem in combinatorial terms. From that point, it remains to check the saturation property for any subset in the system of $T$-weights of a simple $S L(n)$-module. In Section 2 we prove that the saturation property holds for each subset in the set of weights of the representations listed in the Main Theorem. The most powerful methods used here are two criteria given in [15, Thm. 3.5] and [15, Thm. 3.8]. Some reasoning uses the graph theory language. Our reference for graph theory is [7]. In Section 3 we produce non-saturated subsets in sets of weights for all other representations. If the set of weights of the representation with the highest weight $\lambda$ is a subset in the set of weights of the representation with the highest weight $\mu$, and a non-saturated subset for $\lambda$ is known, then one can use it as a non-saturated subset for $\mu$. Fundamental representations form the most difficult class. To work with them, we use the following observation. If a non-saturated subset in the set of weights of the $k$ th fundamental representation of $S L(n)$ is found, then the analogous non-saturated subset exists in the set of weights of the $k$ th fundamental representation of $S L(n+k)$.

Another motivation to study normality of torus orbits' closures comes from a more general problem. Let $G$ be a connected reductive group with a maximal unipotent subgroup $U$ normalized by a maximal torus $T$. Assume that $G$ acts on an affine variety $X$. Then the passage to $U$-invariants allows to reduce the question of normality of a spherical $G$-orbit closure on $X$ to the question of normality of a $T$-orbit closure on $X / / U$, see [1] for a partial realization of this approach.

In further publications, we plan to give a classification of simple $G$-modules with normal $T$-orbit closures for other simple algebraic groups $G$.

\section{Algebraic background and notation}

Let $V$ be a finite-dimensional rational $T$-module. Given any character $\chi$ from the character lattice $\mathfrak{X}(T)$, define a weight subspace $V_{\chi}$ as $V_{\chi}=\{v \in V: t \cdot v=\chi(t) v\}$. It is well known that $V=\bigoplus_{\chi \in \mathfrak{X}(T)} V_{\chi}$, and only finitely many $V_{\chi}$ are nonzero. The set $\left\{\chi_{1}, \chi_{2}, \ldots, \chi_{k}\right\}$ of those $\chi_{i}$ for which $V_{\chi_{i}} \neq 0$ is called the system of weights of the $T$-module $V$. 
Let $\mathbb{Z}_{+}$and $\mathbb{Q}_{+}$denote the sets of integer and rational non-negative numbers, respectively; and let $v_{1}, v_{2}, \ldots, v_{m} \in \mathbb{Q}^{n}$. Consider the semigroup $\mathbb{Z}_{+}\left(v_{1}, v_{2}, \ldots, v_{m}\right)=$ $\left\{n_{1} v_{1}+n_{2} v_{2}+\ldots+n_{m} v_{m}: n_{i} \in \mathbb{Z}_{+}\right\}$, the sublattice $\mathbb{Z}\left(v_{1}, v_{2}, \ldots, v_{m}\right)=\left\{z_{1} v_{1}+\right.$ $\left.z_{2} v_{2}+\ldots+z_{m} v_{m}: z_{i} \in \mathbb{Z}\right\}$, and the rational polyhedral cone $\mathbb{Q}_{+}\left(v_{1}, v_{2}, \ldots, v_{m}\right)=$ $\left\{q_{1} v_{1}+q_{2} v_{2}+\ldots+q_{m} v_{m}: q_{i} \in \mathbb{Q}_{+}\right\}$. Define the following important property of the set $\left\{v_{1}, v_{2}, \ldots, v_{m}\right\}$.

Definition The set of points $\left\{v_{1}, v_{2}, \ldots, v_{m}\right\} \subset \mathbb{Q}^{n}$ is called saturated if

$$
\mathbb{Z}_{+}\left(v_{1}, v_{2}, \ldots, v_{m}\right)=\mathbb{Z}\left(v_{1}, v_{2}, \ldots, v_{m}\right) \cap \mathbb{Q}_{+}\left(v_{1}, v_{2}, \ldots, v_{m}\right) .
$$

The following result provides a well-known combinatorial criterion of normality of the torus orbit closure, see [9]:

Theorem 1.1 Consider a rational linear action of a torus $T$ on a vector space $V$. Let $v \in V$, and $v=v_{\lambda_{1}}+\cdots+v_{\lambda_{s}}, v_{\lambda_{i}} \neq 0$, be its weight decomposition. Then the closure $\overline{T v}$ is normal if and only if the set of characters $\left\{\lambda_{1}, \ldots, \lambda_{s}\right\}$ is saturated.

Definition The set of points $\left\{\lambda_{1}, \lambda_{2}, \ldots, \lambda_{m}\right\} \subset \mathbb{Q}^{n}$ is called hereditary normal if each its subset is saturated.

Corollary 1.2 Given a rational linear action of a torus $T$ on a vector space $V$; let $\left\{\lambda_{1}, \ldots, \lambda_{s}\right\}$ be the set of weights of this action. Then the closure $\overline{T v}$ is normal for each $v \in V$ if and only if $\left\{\lambda_{1}, \ldots, \lambda_{s}\right\}$ is hereditary normal.

Remark The weight system is multiplied by -1 while changing a representation $V$ of the torus $T$ with its dual. Hence the property of normality of all $T$-orbits' closures is preserved.

Let $G=S L(n)$. We fix a maximal torus $T \subset G$ consisting of all diagonal matrices. An element $a=\left(a_{1}, a_{2}, \ldots, a_{n}\right)$ of the lattice $\mathbb{Z}^{n}$ can be interpreted as a character $\chi_{a}$ of the torus $T$ in the following way: $\chi_{a}(t)=t_{1}^{a_{1}} t_{2}^{a_{2}} \ldots t_{n}^{a_{n}}$, where $t=\operatorname{diag}\left(t_{1}, t_{2}, \ldots, t_{n}\right)$. Since $t_{1} t_{2} \ldots t_{n}=1$, the points $a$ and $b$ define the same character if and only if $a-b=\alpha(1,1, \ldots, 1)$. Each $a$ from $\mathbb{Z}^{n}$ has a unique representation $a=\tilde{a}+\alpha(1,1, \ldots, 1)$, where $\tilde{a} \in \mathbb{Q}^{n}, \alpha \in \mathbb{Q}$, and $\sum \tilde{a}_{i}=0$.

Let $\varepsilon_{1}, \varepsilon_{2}, \ldots, \varepsilon_{n}$ be the standard basis of the lattice $\mathbb{Z}^{n}$, and

$$
e_{i}=\widetilde{\varepsilon_{i}}=\left(-\frac{1}{n},-\frac{1}{n}, \ldots,-\frac{1}{n}, \frac{n-1}{i_{\text {th place }}^{n}},-\frac{1}{n}, \ldots,-\frac{1}{n}\right) .
$$

Notice that $e_{1}, e_{2}, \ldots, e_{n}$ (further referred to as a quasi-basis) satisfy the only linear relation

$$
e_{1}+e_{2}+\ldots+e_{n}=0 .
$$

Identify $\mathfrak{X}(T)$ with the $\mathbb{Z}$-lattice generated by $e_{1}, e_{2}, \ldots, e_{n}$. Recall that a weight $\chi_{a}$ is called dominant if and only if $a_{1} \geqslant a_{2} \geqslant \ldots \geqslant a_{n}$. The root lattice for $S L(n)$ is a 
lattice generated by the vectors $e_{1}-e_{2}, e_{2}-e_{3}, \ldots, e_{n-1}-e_{n}$. Due to the ambiguity of notation,

$$
\Phi=\left\{a_{1} e_{1}+a_{2} e_{2}+\ldots+a_{n} e_{n}: n \mid\left(a_{1}+a_{2}+\ldots+a_{n}\right)\right\},
$$

where $\mid$ stands for divisibility, i.e. $n \mid m \Longleftrightarrow \exists z \in \mathbb{Z}, m=n z$.

For a positive integer $s \mid n$, define

$$
\mathbb{Z}_{\equiv 0(s)}\left(e_{1}, \ldots, e_{n}\right)=\left\{\sum_{i=1}^{n} x_{i} e_{i}: x_{i} \in \mathbb{Z}, s \mid \sum_{i=1}^{n} x_{i}\right\}
$$

In this notation the root lattice $\Phi$ coincides with $\mathbb{Z}_{\equiv 0(n)}\left(e_{1}, \ldots, e_{n}\right)$.

Let $V$ be a finite-dimensional simple rational $S L(n)$-module, and $M(V)$ be the system of weights of the module $V$ with respect to the restricted action $T: V$. Introduce a partial order on $M(V): \mu \succeq v$ if and only if for $\xi=\mu-v$ the following conditions hold: $\xi_{1} \geqslant 0, \xi_{1}+\xi_{2} \geqslant 0, \ldots, \xi_{1}+\xi_{2}+\ldots+\xi_{n-1} \geqslant 0$. It is well known that $M(V)$ contains the only maximal element $\lambda$ with respect to $\succeq$, it is called the highest weight of the module. The weight $\lambda$ is dominant; moreover, for any dominant weight $\lambda \in \mathfrak{X}(T)$ there exists a unique simple $S L(n)$-module $V(\lambda)$ with the highest weight $\lambda$ (see [16, Chapter $4, \S 3$, Thm.11] or $[8, \S \S 20-21]$ ). The role of the Weyl group $W$ is played here by the permutation group $S_{n}$, which acts on $\mathbb{Z}^{n}$ by permutations of coordinates. It is known that

$$
M(\lambda):=M(V(\lambda))=\operatorname{conv}\{w \lambda: w \in W\} \cap(\lambda+\Phi),
$$

where $\operatorname{conv}(L)$ denotes the convex hull of the set $L \subset \mathbb{R}^{n}$; see [8, §21.3].

In our situation, Corollary 1.2 can be reformulated in the following way:

Proposition 1.3 Let $V(\lambda)$ be a simple module of a semisimple group $G$ with the highest weight $\lambda$. Then the closure of each $T$-orbit in $V(\lambda)$ is normal if and only if $M(\lambda)$ is hereditary normal.

\section{Positive results}

In this section we prove that certain sets of weights are hereditary normal. First, let us present some machinery which proves that a given set of points $L$ is hereditary normal. Choose a basis in which for any $v \in L$ its coordinates are integer. Represent each point as a column vector of its coordinates in this basis, and let $K=K(L)$ be an integer $p \times q$ matrix formed by all these column vectors. The first algorithm deals with the objects which we had in the very beginning - with the monomial algebras.

Definition To each column $K_{i}=\left(k_{1 i}, \ldots, k_{p i}\right)^{T}$ we put into correspondence a Laurent monomial $t^{K_{i}}=t_{1}^{k_{1 i}} \ldots t_{p}^{k_{p i}}$. The toric ideal $I_{K}$ associated with $K$ is the kernel of the $\mathbb{k}$-algebra homomorphism

$$
\mathbb{C}\left[x_{1}, x_{2}, \ldots, x_{q}\right] \rightarrow \mathbb{C}\left[t_{1}, \ldots, t_{d}, t_{1}^{-1}, \ldots, t_{d}^{-1}\right], x_{i} \mapsto t^{K_{i}} .
$$


Definition Suppose that $u_{+}$and $u_{-}$are two vectors from $\mathbb{Z}_{+}^{p}$ with disjoint supports, and let $f=x^{u_{+}}-x^{u_{-}} \in I_{K}$. We say that $f$ is a circuit in $I_{K}$ if the following two conditions hold:

- all the coordinates of $u^{+}$and $u^{-}$have no common divisor greater than 1;

- the set of variables which actually occur in $f$ is minimal with respect to inclusion among all the binomials of $I_{K}$.

Theorem 2.1 ([15, Thm. 3.8]) A set of points $L$ is hereditary normal if and only if every circuit in $I_{K(L)}$ has at least one square-free monomial.

The next property of $K=K(L)$ deals only with $K$, which makes this method so easy.

Definition The matrix $K$ is called unimodular if it has maximal rank $p$, and all nonzero $p \times p$ minors of $K$ have the same absolute value.

If the rows of $K$ are linearly dependent, then one can omit the redundant rows and check unimodularity for the remaining matrix.

Theorem 2.2 ([15, Thm. 3.5]) If $K(L)$ is unimodular, then $L$ is hereditary normal.

\subsection{The tautological representation}

Its highest weight equals $e_{1}$. The set $M\left(e_{1}\right)$ equals $\left\{e_{1}, \ldots, e_{n}\right\}$. It is unimodular, hence it is hereditary normal.

\subsection{The adjoint representation}

Its highest weight $\lambda$ is equal to $e_{1}-e_{n}$. Acting by $W=S_{n}$, we get all vectors of the form $e_{i}-e_{j}$. Taking the convex hull adds only $\overline{0}$ to this set. We get $M(\lambda)=$ $\{0\} \cup\left\{e_{i}-e_{j}: 1 \leq i, j \leq n, i \neq j\right\}$. It was proved in [15, Ex. 3.7] or [11, Thm.1] that this set is hereditary normal.

\subsection{The representation of $S L(2)$ with the highest weight $3 \pi_{1}$}

Here we have $M(\lambda)=\left\{\left(\frac{3}{2},-\frac{3}{2}\right),\left(\frac{1}{2},-\frac{1}{2}\right),\left(-\frac{1}{2}, \frac{1}{2}\right),\left(-\frac{3}{2}, \frac{3}{2}\right)\right\}$ (in the usual basis). After an appropriate change of basis, $K(M(\lambda))=(3,1,-1,-3)$, the set of all circuits of $I_{K(M(\lambda))}$ is $\left\{x_{1} x_{3}^{3}-1, x_{1} x_{4}-1, x_{1}-x_{2}^{3}, x_{2} x_{3}-1, x_{2}^{3} x_{4}-1, x_{3}^{3}-x_{4}\right\}$. By Theorem 2.1, the set $M(\lambda)$ is hereditary normal.

\subsection{The representation of $S L(2)$ with the highest weight $4 \pi_{1}$}

One has to verify that the set $M(\lambda)=\{(2,-2),(1,-1),(0,0),(-1,1),(-2,2)\}$ is hereditary normal. Here we can make $K(M(\lambda))=(2,1,0,-1,-2)$, the set of all circuits of $I_{K(M(\lambda))}$ is $\left\{x_{1}-x_{2}^{2}, x_{1} x_{4}^{2}-1, x_{1} x_{5}-1, x_{2} x_{4}-1, x_{2}^{2} x_{5}-1, x_{4}^{2}-x_{5}\right\}$. By Theorem 2.1, it is hereditary normal. 
2.5 The representation of $S L(3)$ with the highest weight $2 \pi_{1}$

Its highest weight $\lambda$ is equal to $2 \pi_{1}=2 e_{1}$, and all the weights of this representation are pointed in the figure below.

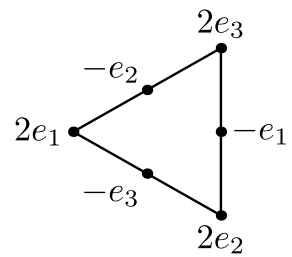

After an appropriate change of basis, $K(M(\lambda))=\left(\begin{array}{cccccc}1 & 0 & -1 & -2 & 0 & 2 \\ 0 & 1 & -1 & 0 & -2 & 2\end{array}\right)$. The set of all circuits of $I_{K(M(\lambda))}$ is $\left\{x_{1} x_{2} x_{3}-1, x_{4} x_{5} x_{6}-1, x_{1}^{2} x_{4}-1, x_{2}^{2} x_{5}-1, x_{3}^{2} x_{6}-\right.$ $\left.1, x_{1}^{2} x_{2}^{2}-x_{6}, x_{1}^{2} x_{3}^{2}-x_{5}, x_{2}^{2} x_{3}^{2}-x_{4}, x_{1}^{2}-x_{4} x_{5}, x_{2}^{2}-x_{4} x_{6}, x_{3}^{2}-x_{5} x_{6}\right\}$. By Theorem 2.1, it is hereditary normal.

2.6 The representation of $S L(6)$ with the highest weight $\pi_{3}$

The highest weight $\lambda$ equals $\left(\frac{1}{2}, \frac{1}{2}, \frac{1}{2},-\frac{1}{2},-\frac{1}{2},-\frac{1}{2}\right), M(\lambda)=\left\{\frac{1}{2}\left(\varepsilon_{1}, \ldots, \varepsilon_{6}\right): \varepsilon_{i}=\right.$ $\left.\pm 1, \sum \varepsilon_{i}=0\right\}$. The corresponding matrix $K$ is $\left(K_{1} \mid-K_{1}\right)$, where

$$
K_{1}=\frac{1}{2}\left(\begin{array}{rrrrrrrrrr}
1 & 1 & 1 & 1 & 1 & 1 & 1 & 1 & 1 & 1 \\
1 & 1 & -1 & 1 & -1 & -1 & 1 & -1 & -1 & -1 \\
1 & -1 & 1 & -1 & 1 & -1 & -1 & 1 & -1 & -1 \\
-1 & 1 & 1 & -1 & -1 & 1 & -1 & -1 & 1 & -1 \\
-1 & -1 & -1 & 1 & 1 & 1 & -1 & -1 & -1 & 1 \\
-1 & -1 & -1 & -1 & -1 & -1 & 1 & 1 & 1 & 1
\end{array}\right)
$$

The rows of $K_{1}$ are linearly dependent. One can exclude the first row, and it remains to check unimodularity of $K_{2}$,

$$
K_{2}=\left(\begin{array}{rrrrrrrrrr}
1 & 1 & -1 & 1 & -1 & -1 & 1 & -1 & -1 & -1 \\
1 & -1 & 1 & -1 & 1 & -1 & -1 & 1 & -1 & -1 \\
-1 & 1 & 1 & -1 & -1 & 1 & -1 & -1 & 1 & -1 \\
-1 & -1 & -1 & 1 & 1 & 1 & -1 & -1 & -1 & 1 \\
-1 & -1 & -1 & -1 & -1 & -1 & 1 & 1 & 1 & 1
\end{array}\right) .
$$

An easy but cumbersome calculation shows that $K_{2}$ is unimodular, all its non-zero minors are equal to \pm 16 , hence $M(\lambda)$ is hereditary normal.

In the next case we use the following lemmas, their proof can be found in [11].

Lemma 2.3 Let $M$ be a non-saturated set and $\alpha$ be a vector such that $\alpha \in M$ and $-\alpha \in M$. Then either $M \backslash\{\alpha\}$ or $M \backslash\{-\alpha\}$ is non-saturated. 
Lemma 2.4 Any set of linearly independent vectors is saturated.

Lemma 2.5 Let $v=q_{1} v_{1}+\cdots+q_{m} v_{m}$, where $v, v_{i}$ are arbitrary vectors, and $q_{i} \in$ $\mathbb{Q}_{+}$. Then one can choose a linearly independent subset $\left\{v_{i_{1}}, \ldots, v_{i_{s}}\right\} \subset\left\{v_{1}, \ldots, v_{m}\right\}$ and numbers $q_{i_{1}}^{\prime}, \ldots, q_{i_{s}}^{\prime} \in \mathbb{Q}_{+}$such that

$$
v=q_{i_{1}}^{\prime} v_{i_{1}}+\cdots+q_{i_{s}}^{\prime} v_{i_{s}} .
$$

Definition We mean by an NSS a non-saturated subset $\left\{v_{1}, v_{2}, \ldots, v_{s}\right\}$ in the set $M$ of weights of a representation. By an ENSS we mean the NSS together with a vector $v$, where $v \in \mathbb{Z}\left(v_{1}, v_{2}, \ldots, v_{m}\right) \cap \mathbb{Q}_{+}\left(v_{1}, v_{2}, \ldots, v_{m}\right)$, and $v \notin \mathbb{Z}_{+}\left(v_{1}, v_{2}, \ldots, v_{m}\right)$.

2.7 The representations of $S L(4), S L(5)$ and $S L(6)$ with the highest weight $\pi_{2}$

The set of weights $M(\lambda)$ is equal to $\left\{e_{i}+e_{j}: 1 \leq i<j \leq n\right\}$, where $n=4,5,6$. Suppose that there exists an $\operatorname{ENSS}\left\{w ; v_{1}, \ldots, v_{m}\right\}, v_{i} \in M(\lambda)$ :

$$
\begin{gathered}
w=z_{1} v_{1}+\cdots+z_{m} v_{m}=q_{1} v_{1}+\cdots+q_{m} v_{m}, \\
z_{i} \in \mathbb{Z}, q_{i} \in \mathbb{Q}_{+}, w \notin \mathbb{Z}_{+}\left(v_{1}, \ldots, v_{m}\right) .
\end{gathered}
$$

Consider all $v_{i}$ occurring in the right side of this equality with a nonzero coefficient $q_{i}$. By Lemma 2.5, we may assume that they are linearly independent. To simplify the reasoning, consider vector $v=w-\left\lfloor q_{1}\right\rfloor v_{1}-\cdots-\left\lfloor q_{m}\right\rfloor v_{m}$ instead of $w$. It is easy to see that $v$ belongs to $\mathbb{Z}\left(v_{1}, \ldots, v_{m}\right)$, to $\mathbb{Q}_{+}\left(v_{1}, \ldots, v_{m}\right)$ and does not belong to $\mathbb{Z}_{+}\left(v_{1}, \ldots, v_{m}\right)$. We yield that $\left\{v ; v_{1}, \ldots, v_{m}\right\}$ is also an ENSS. After this change all the coefficients of the $\mathbb{Q}_{+}$-combination belong to $[0,1)$.

Let $\Gamma$ be the quiver associated with $\left\{v_{1}, \ldots, v_{m}\right\}$. Construct a subgraph $\Gamma^{\prime} \subset \Gamma$ : take all the vertices of $\Gamma$ and all the edges of $\Gamma$ entering into the $\mathbb{Q}_{+}$-combination above with nonzero coefficients. Write the coefficients of the $\mathbb{Q}_{+}$-combination at the edges of $\Gamma^{\prime}$. The further proof consists of a search of all possible graphs $\Gamma^{\prime}$. The following observations simplify the search.

(0.1) The number of edges in each connected component of $\Gamma^{\prime}$ is not greater than the number of vertices. Otherwise, the vectors corresponding to the edges of this component are linearly dependent.

(0.2) The number of edges in $\Gamma^{\prime}$ is less than the number of vertices (it follows from $(*)$ that the dimension of the enveloping space equals $n-1)$.

(0.3) The graph $\Gamma^{\prime}$ does not contain even cycles. It follows from the fact that the edges of an even cycle are linearly dependent: their alternating sum is zero.

(0.4) It follows from (0.1) that each connected component of $\Gamma^{\prime}$ either is a tree or contains exactly one cycle. In the second case it follows from $(0.3)$ that this cycle is odd.

(0.5) It follows from (0.2) that $\Gamma^{\prime}$ has a vertex of degree 0 or 1 .

At each vertex, count the sum of all coefficients on the incident edges, and for each sum take its fractional part. All these fractional parts are equal due to the fact that all the sums in vertices (they equal the coordinates of $v$ ) become integer after subtracting $(*)$ with an appropriate coefficient. Now we conclude that 
Fig. 1 Graph $\Gamma^{\prime}$

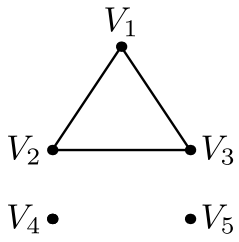

(0.6) $\Gamma^{\prime}$ does not contain vertices of degree 0 and 1 simultaneously: if it does, the fractional parts of the sums in vertices are all equal to 0 , but in the terminal vertex this sum has only one summand and is not an integer. We consider these two cases independently.

Case 1. Graph $\Gamma^{\prime}$ has a vertex of degree 0 .

(1.1) Any other connected component of $\Gamma^{\prime}$ is either a point or has no terminal vertices (it follows from (0.6)). Moreover, it follows from (0.4) that it is an odd cycle.

(1.2) We have $n \leq 6$, consequently, the number of edges in $\Gamma^{\prime}$ is $\leq 5$, but any odd cycle has $\geq 3$ edges, and we yield that $\Gamma^{\prime}$ has at most one cycle.

Fulfill an exhaustive search within all graphs $\Gamma^{\prime}$ having a vertex of degree 0 :

$n=4$, graph is a cycle of length 3 and a point,

$n=5, \quad$ graph is a cycle of length 3 and two points,

$n=6, \quad$ graph is a cycle of length 3 and three points,

$n=6, \quad$ graph is a cycle of length 5 and a point.

The only possible $\mathbb{Q}_{+}$-combination in these cases is $\frac{1}{2}\left(v_{1}+\cdots+v_{s}\right)$. This means that $v=e_{1}+e_{2}+\ldots+e_{s}, s \in\{3,5\}, e_{1}, \ldots, e_{s}$ correspond to the vertices of the cycle. But it does not lie in $\mathbb{Z}\left(v_{1}, \ldots, v_{m}\right)$ when $n$ is even. When $n=5$, consider also the graph $\Gamma$. Since $v$ is a $\mathbb{Z}$-combination of the edges of $\Gamma, \Gamma$ has more than 3 edges: $\Gamma \supset \Gamma^{\prime}, \Gamma \neq \Gamma^{\prime}$ and $\Gamma^{\prime}$ has 3 edges. In the representation $e_{1}+e_{2}+\ldots+e_{s}$ the sum of coefficients of $v$ is odd, hence we should apply $(*)$ to the existing $\mathbb{Z}$-combination to get the representation $e_{1}+e_{2}+\ldots+e_{s}$. For this purpose the edges of $\Gamma$ should touch all the vertices of $\Gamma$ (we name this property $(* *)$ ).

In Fig. 1 the graph $\Gamma^{\prime}$ is drawn. To satisfy $(* *), \Gamma$ should contain at least the following edges (up to symmetry): see Fig. 2a, 2b or 2c. The vertices corresponding to $e_{i}$ are called $V_{i}$. But in all cases we get a contradiction, since $e_{1}+e_{2}+e_{3}$ is already a $\mathbb{Z}_{+}$-combination:

$\begin{array}{ll}\text { in Fig. 2a: } & e_{1}+e_{2}+e_{3}=V_{1} V_{2}+V_{2} V_{3}+V_{1} V_{3}+V_{4} V_{5}, \\ \text { in Fig. 2b: } & e_{1}+e_{2}+e_{3}=V_{4} V_{2}+V_{2} V_{1}+V_{1} V_{3}+V_{3} V_{5}, \\ \text { in Fig. 2c: } & e_{1}+e_{2}+e_{3}=V_{4} V_{2}+V_{2} V_{5}+2 V_{1} V_{3} .\end{array}$

We have shown that the graph $\Gamma$ does not provide an NSS if $\Gamma^{\prime}$ has a vertex of degree 0 .

Case 2. The graph $\Gamma^{\prime}$ has a vertex $X$ of degree 1 . Let $X Y$ be an edge incident to $X$. We need to subtract $(*)$ with the same multiplicity as at $X Y$. We get: 


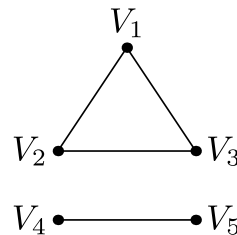

(a)

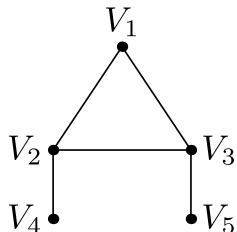

(b)

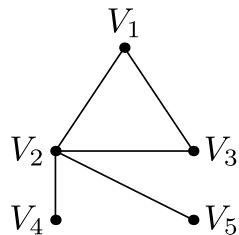

(c)

Fig. 2 Graph $\Gamma$ should contain one of these graphs as a subgraph

(2.1) Since $X$ is a terminal vertex of $\Gamma^{\prime}$, either $X Y$ is a connected component of $\Gamma^{\prime}$ or the degree of $Y$ is $\geq 3$. Indeed, suppose that the degree of $Y$ is 2 . Let $Y Z$ be the second edge incident to $Y$, and let $q$ be the value written at $Y Z$. After subtracting $(*)$ the coefficient at $Y$ becomes equal to $q$, but it should be integer, and we know that $q \in(0,1)$. This is a contradiction.

Find all possible connected components of $\Gamma^{\prime}$.

On 2 vertices:

On 3 vertices:

On 4 vertices: $\dot{V}$ and $\mathscr{Z}$

If $\Gamma^{\prime}$ has a connected component on 5 or 6 vertices, then this component coincides with $\Gamma^{\prime}$. Using this observation together with $(0.2)$, we obtain that $\Gamma^{\prime}$ is a tree. Taking into account (2.1), it remains to consider only the following trees: $\bullet \cdot, \cdot$. and But the edges of are linearly dependent (when $n=6$, one should sum all the thin edges, then subtract the thick one, and obtain $(*))$. Therefore, this graph should not be considered. The result is

On 5 vertices:

On 6 vertices:

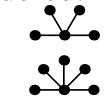

Fulfill an exhaustive search within all graphs $\Gamma^{\prime}$ on $n$ vertices satisfying all the conditions above. In the case when one of the connected components of $\Gamma^{\prime}$ is a claw (i.e., all the edges are incident to one vertex) with at least 4 vertices, its central vertex will correspond to $e_{1}$ (it is easy to see that $\Gamma^{\prime}$ cannot have more than one claw).

\begin{tabular}{|c|c|c|}
\hline$n$ & $\begin{array}{c}\text { Splitting into } \\
\text { connected components }\end{array}$ & $\begin{array}{c}\text { Admissible } \\
\text { graphs }\end{array}$ \\
\hline 4 & $2+2$ & \\
\hline 4 & 4 & \\
\hline 5 & $2+3$ & \\
\hline 5 & 5 & \\
\hline 6 & $2+2+2$ & \\
\hline 6 & $2+4$ & \\
\hline 6 & 6 & .2 \\
\hline
\end{tabular}

The graphs $\lfloor$ and $\stackrel{Z}{\longrightarrow}$ do not satisfy our conditions: their edges are linearly dependent. 
If we start with $\mathscr{K}$, we can obtain only $e_{1}$ as the $\mathbb{Q}_{+}$-combination: all the three edges should appear in the $\mathbb{Q}_{+}$-combination with the same coefficient, say $a$, $a \in(0,1)$. We sum these three vectors, obtain $3 a e_{1}+a e_{2}+a e_{3}+a e_{4}$, and subtract $(*)$ with an appropriate coefficient. Finally we obtain $2 a e_{1}$. In this notation it already has integer coordinates (equal to zero), this means that all the other coordinates, $2 a$ among them, should be integers, $a=\frac{1}{2}, v=e_{1}$. But $v$ cannot be obtained as a $\mathbb{Z}$-combination of vectors of type $e_{i}+e_{j}$. Indeed, each $v_{i}$ has an even sum of coordinates, $n$ is even, subtracting $(*)$ with an integer coefficient does not change parity of the sum of coordinates, and this proves that any vector from $\mathbb{Z}\left\{v_{i}\right\}_{i=1}^{m}$ has even sum of coordinates.

The edges of the graph $\lfloor\triangle$ are linearly dependent (here $n=5$ ) because (2.first edge + the sum of the edges of the cycle) $=0$.

The graph. $\mathcal{V}$ : using similar reasoning, $v=e_{1}$ or $2 e_{1}$. But there exists an edge in $\Gamma \backslash \Gamma^{\prime}$, hence $e_{1}$ is a $\mathbb{Z}_{+}$-combination of the edges of $\Gamma$ : take the sum of thick edges of $\mathbf{N}$.

The edges of $\bullet:$ and $\bullet:$ are linearly dependent.

In the graph $\stackrel{L}{L}$ the vector $v$ may be equal only to $e_{1}$, but $e_{1}$ can not be obtained as a $\mathbb{Z}$-combination: 6 is even, and the sum of coordinates of $e_{1}$ is odd.

In the graph $\because$. the vector $v$ has to be proportional to $e_{1}$, moreover, the coefficient should be even (we use the reasoning as above, from the fact that 6 is even it follows that the sum of coordinates is even for any vector from $\left.\mathbb{Z}\left(v_{1}, \ldots, v_{m}\right)\right)$. But if we add any edge to this set, $2 e_{1}$ will be obtained as a $\mathbb{Z}_{+}$-combination: take the sum of thick edges of $\mathbf{A}$.

All the cases are considered, and this completes the proof.

\section{Negative results}

Let $\lambda$ be a highest weight not listed in the Main Theorem. One has to construct an NSS in $M(\lambda)$. There are two possibilities for $\lambda$ : either the absolute values of all its usual coordinates are $<1$, or $\lambda$ has a coordinate with the absolute value $\geqslant 1$. Speaking informally, the second case is practically always the consequence of the first one (Lemma 3.10), but the NSS in the first case is constructed recursively and its capacity increases when $n$ increases. The construction of the second case gives an NSS of only 4 vectors for any $n$.

To prove that a set $\left\{v_{1}, \ldots, v_{m}\right\}$ is not saturated, we construct a so-called discriminating function $f(v)$ with the following properties: linearity and $f\left(v_{i}\right)>0$, $i=1,2, \ldots, m$. The discriminating function will be applied as follows. If one wants to show that $\left\{v_{0} ; v_{1}, \ldots, v_{m}\right\}$ is an ENSS, it suffices to present the corresponding $\mathbb{Q}_{+}$and $\mathbb{Z}$-combinations for $v_{0}$ and construct a discriminating function $f$, such that $f\left(v_{0}\right)$ cannot be composed as the sum of $f\left(v_{i}\right)$ with $\mathbb{Z}_{+}$-coefficients.

By $x_{i}$ we denote the $i$ th quasi-coordinate of a vector, if its quasi-basis representation is fixed. Note that $f=a_{1} x_{1}+\ldots+a_{n} x_{n}$ in quasi-basis is well-defined if and only if $a_{1}+\ldots+a_{n}=0$. 


\subsection{The fundamental weights}

In this case $\lambda$ equals

$$
\pi_{k}=\pi_{k, n}=\left(\frac{n-k}{n}, \ldots, \frac{n-k}{n},-\frac{k}{n}, \ldots,-\frac{k}{n}\right)
$$

in the usual basis, $0<k<n, n \geqslant 3$ (if $n=2$, the corresponding representation is mentioned in the Main Theorem). In some proofs we consider $\pi_{k}$ for $S L(n)$ 's of different dimensions simultaneously, so the second index in the notation $\pi_{k, n}$ carries this data. Here $M(\lambda)=\left\{\sigma \lambda: \sigma \in S_{n}\right\}$. The highest weight is equal to $e_{1}+e_{2}+\ldots+e_{k}$ in quasi-basis, all the points of $M(\lambda)$ have form $e_{i_{1}}+e_{i_{2}}+\ldots+e_{i_{k}}, 1 \leq i_{1}<i_{2}<$ $\ldots<i_{k} \leq n$.

Now we can reformulate the problem. Let $\left\{e_{i}\right\}$ be the quasi-basis, $k<n$, and the weight $\lambda=\pi_{k}$ is not listed in the Main Theorem. One has to find a non-saturated subset in the set

$$
\left\{e_{i_{1}}+e_{i_{2}}+\ldots+e_{i_{k}}: 1 \leq i_{1}<i_{2}<\ldots<i_{k} \leq n\right\} .
$$

The construction uses induction on $n$. In the next section we produce the NSSes which are the base of induction.

\subsubsection{Important particular cases}

Example $3.1 n=7, k=2$. The NSS consists of those and only those vectors which are the sums of two quasi-basis vectors connected with an edge in the graph below. We have

$$
\begin{aligned}
v & =e_{1}+e_{2}+e_{3}=\frac{1}{2}\left(\left(e_{1}+e_{2}\right)+\left(e_{2}+e_{3}\right)+\left(e_{1}+e_{3}\right)\right), \\
v & =-\left(e_{4}+e_{5}+e_{6}+e_{7}\right) \\
& =2\left(e_{2}+e_{3}\right)-\left(e_{2}+e_{4}\right)-\left(e_{2}+e_{5}\right)-\left(e_{3}+e_{6}\right)-\left(e_{3}+e_{7}\right) .
\end{aligned}
$$

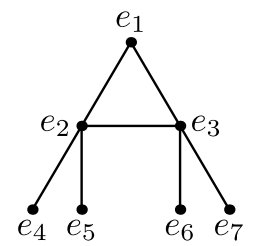

Let $f=5\left(x_{2}+x_{3}\right)-2\left(x_{1}+x_{4}+x_{5}+x_{6}+x_{7}\right)$. Then

$$
\begin{aligned}
f\left(e_{1}+e_{2}\right) & =f\left(e_{1}+e_{3}\right)=f\left(e_{2}+e_{4}\right)=f\left(e_{2}+e_{5}\right)=f\left(e_{3}+e_{6}\right) \\
& =f\left(e_{3}+e_{7}\right)=3,
\end{aligned}
$$




$$
f\left(e_{2}+e_{3}\right)=10, f(v)=f\left(e_{1}+e_{2}+e_{3}\right)=5 \cdot 2-2=8 .
$$

It is clear that 8 cannot be represented as a sum where each summand equals either 3 or 10 .

Example $3.2 n=8, k=3$. Consider the following vectors (in quasi-basis):

$$
\left(\begin{array}{l}
v_{1} \\
v_{2} \\
v_{3} \\
v_{4} \\
v_{5} \\
v_{6} \\
v_{7} \\
v_{8}
\end{array}\right)=\left(\begin{array}{llllllll}
0 & 0 & 1 & 1 & 1 & 0 & 0 & 0 \\
1 & 0 & 0 & 1 & 1 & 0 & 0 & 0 \\
1 & 1 & 0 & 0 & 1 & 0 & 0 & 0 \\
1 & 1 & 1 & 0 & 0 & 0 & 0 & 0 \\
0 & 1 & 1 & 1 & 0 & 0 & 0 & 0 \\
0 & 0 & 1 & 1 & 0 & 1 & 0 & 0 \\
0 & 1 & 0 & 1 & 0 & 0 & 1 & 0 \\
0 & 1 & 1 & 0 & 0 & 0 & 0 & 1
\end{array}\right)
$$

Take $v=(1,1,1,1,1,0,0,0)=\frac{1}{3}\left(v_{1}+v_{2}+v_{3}+v_{4}+v_{5}\right)=2 v_{5}-v_{6}-v_{7}-v_{8}$. Let $f=x_{1}+5\left(x_{2}+x_{3}+x_{4}\right)+2 x_{5}-6\left(x_{6}+x_{7}+x_{8}\right)$. Then

$$
\begin{gathered}
f\left(v_{1}\right)=12, \quad f\left(v_{2}\right)=f\left(v_{3}\right)=8, \quad f\left(v_{4}\right)=11, \\
f\left(v_{5}\right)=15, \quad f\left(v_{6}\right)=f\left(v_{7}\right)=f\left(v_{8}\right)=4, \quad f(v)=18 .
\end{gathered}
$$

It is easy to see that 18 cannot be represented as a sum of $4,8,11,12$, or 15 .

Example $3.3 n=2 k, k \geqslant 4$.

$$
\left(\begin{array}{c}
v_{1} \\
v_{2} \\
v_{3} \\
\vdots \\
v_{k-1} \\
v_{k} \\
v_{k+1} \\
v_{k+2}
\end{array}\right)=\left(\begin{array}{cccccccccccc}
1 & 0 & 0 & \ldots & 0 & 0 & 0 & 1 & 1 & \ldots & 1 & 1 \\
0 & 1 & 0 & \ldots & 0 & 0 & 1 & 0 & 1 & \ldots & 1 & 1 \\
0 & 0 & 1 & \ldots & 0 & 0 & 1 & 1 & 0 & \ldots & 1 & 1 \\
\vdots & \vdots & \vdots & \ddots & \vdots & \vdots & \vdots & \vdots & \vdots & \ddots & \ddots & \vdots \\
0 & 0 & 0 & \ldots & 1 & 0 & 1 & 1 & 1 & \ldots & 0 & 1 \\
0 & 0 & 0 & \ldots & 0 & 1 & 1 & 1 & 1 & \ldots & 1 & 0 \\
0 & 1 & 0 & \ldots & 0 & 0 & 1 & 1 & 1 & \ldots & 1 & 0 \\
1 & 1 & 0 & \ldots & 0 & 0 & 0 & 1 & 1 & \ldots & 1 & 0
\end{array}\right)
$$

Let us show that it is an NSS. Set $v=(\underbrace{0, \ldots, 0}_{k}, \underbrace{1, \ldots, 1}_{k}), v=v_{1}+v_{k+1}-v_{k+2}$,

$$
\begin{aligned}
& \frac{1}{k-2}\left(v_{1}+\cdots+\right.\left.v_{k}\right)=\frac{1}{k-2}(\underbrace{1, \ldots, 1}_{k}, \underbrace{k-1, \ldots, k-1}_{k})= \\
&=\frac{1}{k-2}(\underbrace{0, \ldots, 0}_{k}, \underbrace{k-2, \ldots, k-2}_{k})=(\underbrace{0, \ldots, 0}_{k}, \underbrace{1, \ldots, 1}_{k})=v .
\end{aligned}
$$


To explain why $v$ is not a $\mathbb{Z}_{+}$-combination of vectors $v_{i}$, consider two cases.

Case 1: $k=4$. Let $f=-6 x_{3}-7 x_{4}+5\left(x_{5}+x_{6}+x_{7}\right)-2 x_{8}$. Then

$$
f\left(v_{1}\right)=f\left(v_{2}\right)=8, f\left(v_{3}\right)=2, f\left(v_{4}\right)=8, f\left(v_{5}\right)=15, f\left(v_{6}\right)=10, f(v)=13 .
$$

But it is easy to see that 13 cannot be represented as a sum of $2,8,10$, or 15 .

Case $2: k \geqslant 5$. Let $f=(k-2)\left(x_{k+1}+\cdots+x_{2 k}\right)-k\left(x_{3}+\cdots+x_{k}\right)$. Then

$$
\begin{gathered}
f\left(v_{1}\right)=f\left(v_{2}\right)=(k-2)(k-1), \\
f\left(v_{3}\right)=f\left(v_{4}\right)=\cdots=f\left(v_{k}\right)=(k-1)(k-2)-k, \\
f\left(v_{k+1}\right)=(k-2)(k-1), \quad f\left(v_{k+2}\right)=(k-2)^{2}, \quad f(v)=k(k-2) .
\end{gathered}
$$

If $k \geqslant 6$, then two least possible summands give too much: $2((k-1)(k-2)-k)>$ $k(k-2)$, if $k=5$, then 15 should be represented as a sum of 12,7 , or 9 , but this is impossible.

Example $3.4 n=2 k+1, k \geqslant 3$.

$$
\left(\begin{array}{c}
v_{1} \\
v_{2} \\
v_{3} \\
\vdots \\
v_{k} \\
v_{k+1} \\
v_{k+2} \\
v_{k+3} \\
\vdots \\
v_{2 k} \\
v_{2 k+1}
\end{array}\right)=\left(\begin{array}{ccccccccccc}
0 & 1 & 1 & \ldots & 1 & 1 & 0 & 0 & \ldots & 0 & 0 \\
1 & 0 & 1 & \ldots & 1 & 1 & 0 & 0 & \ldots & 0 & 0 \\
1 & 1 & 0 & \ldots & 1 & 1 & 0 & 0 & \ldots & 0 & 0 \\
\vdots & \vdots & \vdots & \ddots & \vdots & \vdots & \vdots & \vdots & \ddots & \vdots & \vdots \\
1 & 1 & 1 & \ldots & 0 & 1 & 0 & 0 & \ldots & 0 & 0 \\
1 & 1 & 1 & \ldots & 1 & 0 & 0 & 0 & \ldots & 0 & 0 \\
0 & 1 & \ldots & 1 & 1 & 0 & 1 & 0 & \ldots & 0 & 0 \\
1 & 0 & \ldots & 1 & 1 & 0 & 0 & 1 & \ldots & 0 & 0 \\
\vdots & \vdots & \ddots & \vdots & \vdots & \vdots & \vdots & \vdots & \ddots & \vdots & \vdots \\
1 & 1 & \ldots & 0 & 1 & 0 & 0 & 0 & \ldots & 1 & 0 \\
1 & 1 & \ldots & 1 & 0 & 0 & 0 & 0 & \ldots & 0 & 1
\end{array}\right)
$$

$$
\begin{aligned}
& \text { Let } v=(\underbrace{1, \ldots, 1}_{k+1}, \underbrace{0, \ldots, 0}_{k}) \text {. Then } \\
& v=\frac{1}{k}\left(v_{1}+\cdots+v_{k+1}\right)=\frac{1}{k}(\underbrace{k, \ldots, k}_{k+1}, \underbrace{0, \ldots, 0}_{k})=(\underbrace{1, \ldots, 1}_{k+1}, \underbrace{0, \ldots, 0}_{k}), \\
& (k-1) v_{k+1}-v_{k+2}-\cdots-v_{2 k+1}= \\
& =(k-1)(\underbrace{1, \ldots, 1}_{k}, \underbrace{0, \ldots, 0}_{k+1})-(\underbrace{k-1, \ldots, k-1}_{k}, 0, \underbrace{1, \ldots, 1}_{k})=
\end{aligned}
$$




$$
=(\underbrace{0, \ldots, 0}_{k+1}, \underbrace{-1, \ldots,-1}_{k})=(\underbrace{1, \ldots, 1}_{k+1}, \underbrace{0, \ldots, 0}_{k})=v .
$$

It suffices to show that $v$ does not belong to $\mathbb{Z}_{+}\left(v_{1}, v_{2}, \ldots, v_{2 k+1}\right)$. Let $f=(k+$ 1) $\left(x_{1}+\cdots+x_{k}\right)-k\left(x_{k+1}+\cdots+x_{2 k+1}\right)$. Then

$$
\begin{gathered}
f\left(v_{1}\right)=\cdots=f\left(v_{k}\right)=k^{2}-k-1, \quad f\left(v_{k+1}\right)=k(k+1), \\
f\left(v_{k+2}\right)=\cdots=f\left(v_{2 k+1}\right)=k^{2}-k-1, \quad f(v)=k^{2} .
\end{gathered}
$$

But if $k \geqslant 3$, then $k^{2}<2\left(k^{2}-k-1\right)$, so $k^{2}$ cannot be represented as a sum where each summand equals either $\left(k^{2}-k-1\right)$ or $k(k+1)$. This means that $v \notin \mathbb{Z}_{+}\left(v_{1}, v_{2}, \ldots, v_{2 k+1}\right)$.

Example $3.5 n=8, k=2$.

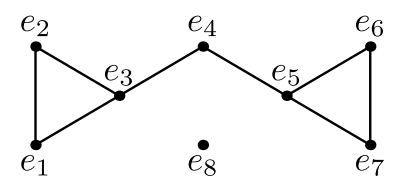

The NSS consists of vectors which are sums of two quasi-basis vectors connected with an edge in the graph above. Let $v=e_{1}+e_{2}+e_{3}+e_{5}+e_{6}+e_{7}$. Then

$$
\begin{gathered}
v=\frac{1}{2}\left(\left(e_{1}+e_{2}\right)+\left(e_{2}+e_{3}\right)+\left(e_{1}+e_{3}\right)+\left(e_{5}+e_{6}\right)+\left(e_{6}+e_{7}\right)+\left(e_{5}+e_{7}\right)\right), \\
v=\left(e_{1}+e_{2}\right)+\left(e_{3}+e_{4}\right)-\left(e_{4}+e_{5}\right)+\left(e_{5}+e_{6}\right)+\left(e_{5}+e_{7}\right) .
\end{gathered}
$$

Check that $e_{1}+e_{2}+e_{3}+e_{5}+e_{6}+e_{7}$ cannot be represented as a $\mathbb{Z}_{+}$-combination of the vectors of our set. Let $f=x_{1}+x_{2}+x_{3}+2\left(x_{5}+x_{6}+x_{7}\right)+9 x_{4}-18 x_{8}$. Then

$$
\begin{gathered}
f\left(e_{1}+e_{2}\right)=f\left(e_{2}+e_{3}\right)=f\left(e_{1}+e_{3}\right)=2, \\
f\left(e_{5}+e_{6}\right)=f\left(e_{6}+e_{7}\right)=f\left(e_{5}+e_{7}\right)=4, \\
f\left(e_{3}+e_{4}\right)=10, \quad f\left(e_{4}+e_{5}\right)=11, \quad f(v)=9 .
\end{gathered}
$$

But 9 cannot be represented as the sum of integers $2,4,10$, or 11 .

Example $3.6 n=9, k=3$. Consider the following vectors:

$$
\begin{array}{ll}
v_{1}=e_{1}+e_{2}+e_{4}, & v_{5}=e_{1}+e_{3}+e_{8}, \\
v_{2}=e_{1}+e_{2}+e_{5}, & v_{6}=e_{1}+e_{3}+e_{9}, \\
v_{3}=e_{2}+e_{3}+e_{6}, & v_{7}=e_{2}+e_{4}+e_{6} .
\end{array}
$$




$$
v_{4}=e_{2}+e_{3}+e_{7}
$$

Then $v=e_{1}+e_{2}+e_{3}=\frac{1}{3}\left(v_{1}+v_{2}+v_{3}+v_{4}+v_{5}+v_{6}\right)=v_{1}+v_{3}-v_{7}$.

Check that $v$ is not a $\mathbb{Z}_{+}$-combination of $v_{1}, v_{2}, v_{3}, v_{4}, v_{5}, v_{6}$, and $v_{7}$. Let $f=$ $5\left(x_{1}+x_{2}+x_{3}+x_{4}\right)-4\left(x_{5}+x_{6}+x_{7}+x_{8}+x_{9}\right)$. Then $f\left(v_{1}\right)=15$,

$$
f\left(v_{2}\right)=f\left(v_{3}\right)=f\left(v_{4}\right)=f\left(v_{5}\right)=f\left(v_{6}\right)=f\left(v_{7}\right)=6, f(v)=15 .
$$

Note that $v \neq v_{1}$ and $f\left(v_{1}\right)=f(v)$, so we conclude that if $v \in \mathbb{Z}_{+}\left(v_{1}, \ldots, v_{7}\right)$, then $v_{1}$ does not occur in this decomposition. But $6 \nmid 15$, and this means that $v$ cannot be obtained as a $\mathbb{Z}_{+}$-combination of $v_{i}$ 's.

Example $3.7 n=10, k=4$. Consider the following vectors:

$$
\begin{array}{ll}
v_{1}=e_{1}+e_{2}+e_{3}+e_{5}, & v_{4}=e_{5}+e_{6}+e_{7}+e_{8}, \\
v_{2}=e_{1}+e_{2}+e_{4}+e_{6}, & v_{5}=e_{5}+e_{7}+e_{8}+e_{9}, \\
v_{3}=e_{3}+e_{4}+e_{5}+e_{6}, & v_{6}=e_{6}+e_{7}+e_{8}+e_{10}, \\
v=e_{1}+e_{2}+e_{3}+e_{4}+e_{5}+e_{6}=\frac{1}{2}\left(v_{1}+v_{2}+v_{3}\right)=v_{4}-v_{5}-v_{6} .
\end{array}
$$

Let us show that $v \notin \mathbb{Z}_{+}\left(v_{1}, \ldots, v_{6}\right)$. Let $f=x_{1}+x_{3}+x_{4}+6 x_{7}+6 x_{8}-7 x_{9}-$ $8 x_{10}$. Then

$$
f\left(v_{1}\right)=f\left(v_{2}\right)=f\left(v_{3}\right)=2, f\left(v_{4}\right)=12, f\left(v_{5}\right)=5, f\left(v_{6}\right)=4, f(v)=3 .
$$

But it is clear that 3 cannot be represented as a sum of $2,4,5$, or 12 .

\subsubsection{Case when $k \nmid n,(n-k) \nmid n$}

It follows that $n \geqslant 5$. The exceptional case $\frac{k}{n} \in\left\{\frac{2}{5}, \frac{3}{5}\right\}$ will be considered at the end of the section. Below we suppose that $\frac{k}{n} \notin\left\{\frac{2}{5}, \frac{3}{5}\right\}$, which gives $n \geqslant 7$.

Lemma 3.8 Assume that there exists an NSS for a pair $(n, k)$, where $(n, k)$ satisfies the conditions above. Then for each $r \in \mathbb{N}$, there exists an NSS for the pair $(n r, k r)$.

Proof Consider an arbitrary vector from $M\left(\pi_{k, n}\right)$. Write down its quasi-coordinates $r$ times in succession. The result is a vector from $M\left(\pi_{k r, n r}\right)$ : it has $k r 1$ 's and $(n-k) r$ 0 's. If one takes an NSS for $(n, k)$ and performs this procedure on each vector, the result will be an NSS for $(n r, k r)$.

Thus, if we construct an NSS for all pairs $(n, k)$ where $\operatorname{gcd}(n, k)=1$, then the NSS for all other pairs will be constructed according to Lemma 3.8.

Lemma 3.9 (The Step procedure) If there exists an NSS for a pair $(n, k)$, then there exists an NSS for the pair $(n+k, k)$. 
Proof The keypoint is that if one takes a weight from $M\left(\pi_{k, n}\right)$, writes it down in the form where all quasi-coordinates are equal to 0 or to 1 , and adds $k$ coordinates equal to 0 , then this weight can be considered as a weight from $M\left(\pi_{k, n+k}\right)$. If one starts with an ENSS $\left(v ; v_{1}, v_{2}, \ldots, v_{m}\right)$ for $(n, k)$, then one should perform this procedure on all its vectors and after that add one more vector $v_{m+1}$ which has 0 s at the first $n$ positions and $1 \mathrm{~s}$ at the $k$ adjoint positions. Now we show that the obtained set $\left(v^{\prime} ; v_{1}^{\prime}, \ldots, v_{m}^{\prime}, v_{m+1}\right)$ is indeed an NSS in $M\left(\pi_{k, n+k}\right)$.

Suppose that a vector $v$ lies in the ENSS for $(n, k), v=q_{1} v_{1}+q_{2} v_{2}+\ldots+q_{s} v_{s}$, $q_{i} \in \mathbb{Q}_{+}$. If we fix some representations in quasi-basis for $v$ and for all $v_{i}$, then this equality can be re-written in the formal basis in the following form:

$$
v=q_{1} v_{1}+q_{2} v_{2}+\ldots+q_{s} v_{s}-\alpha\left(f_{1}+f_{2}+\ldots+f_{n}\right),
$$

where $f_{i}$ 's are the preimages of $e_{i}$ 's under the projection $\mathbb{Q}^{n} \rightarrow \mathfrak{X}(T) \otimes_{\mathbb{Z}} \mathbb{Q}$, $\left(q_{1}, q_{2}, \ldots, q_{n}\right) \mapsto q_{1} e_{1}+q_{2} e_{2}+\ldots+q_{n} e_{n}$. Obviously, $f_{i}$ 's are linearly independent. For each $v_{i}$, fix a representation in which it has $k$ coordinates 1 and $n-k$ coordinates 0 . The vector $v$ is nonzero, consequently, it has a representation where all coordinates are nonnegative, but some of them are zeroes. Fix this representation. Then $\alpha \geq 0$ (otherwise all coordinates of $v$ are strictly positive), and we get that in $\mathbb{Q}^{n+k}$ the following equality holds:

$$
v^{\prime}=q_{1} v_{1}^{\prime}+q_{2} v_{2}^{\prime}+\ldots+q_{s} v_{s}^{\prime}+\alpha\left(f_{n+1}+\cdots+f_{n+k}\right)-\alpha\left(f_{1}+f_{2}+\ldots+f_{n+k}\right) .
$$

This shows that $v^{\prime}$ lies in the $\mathbb{Q}_{+}$-cone generated by $v_{1}^{\prime}, \ldots, v_{m}^{\prime}, v_{m+1}$ (here all vectors taken in quasi-basis $\left.\left\{e_{1}, \ldots, e_{n+k}\right\}\right)$.

Similarly one can show that $v^{\prime}$ lies in the $\mathbb{Z}\left(v_{1}^{\prime}, \ldots, v_{m}^{\prime}, v_{m+1}\right)$.

To prove that the constructed set is an ENSS, it remains to show that $v^{\prime}$ does not lie in $\mathbb{Z}_{+}\left(v_{1}^{\prime}, \ldots, v_{m}^{\prime}, v_{m+1}\right)$. Suppose the contrary. Let $v^{\prime} \in \mathbb{Z}_{+}\left(v_{1}^{\prime}, \ldots, v_{m}^{\prime}, v_{m+1}\right)$. Omit last $k$ coordinates. We get that $v \in \mathbb{Z}_{+}\left(v_{1}, \ldots, v_{m}\right)$, so $\left\{v ; v_{1}, v_{2}, \ldots, v_{m}\right\}$ is not an $\operatorname{ENSS}$ for $(n, k)$.

Notice that this proof also shows how to construct an NSS for a pair $(n+k, k)$ if the NSS for the pair $(n, k)$ is given.

Now we can explain how, using these Lemmas, the NSS's can be constructed for all pairs $(n, k)$, for which the following three conditions hold:

(1) $1<k<n-1$,

(2) $\operatorname{gcd}(n, k)=1$,

(3) $n \geqslant 7$.

Use descent on $n$. Suppose the NSSes are constructed for all pairs $(m, l)$ with $m<n$, satisfying the conditions above. Take a pair $(n, k)$. Suppose $k<\frac{n}{2}$ (otherwise change it by $n-k$ and seek for an NSS for the pair $(n, n-k)$, the case $n=2 k$ is impossible because $\operatorname{gcd}(n, k)=1)$. If all the conditions are held for the pair $(n-k, k)$, then we have an NSS for it, and using the Step procedure, this NSS can be remade into the NSS for $(n, k)$. Let us find all the cases when at least one of the conditions fails for $(n-k, k)$.

Condition (1) fails iff $n=2 k+1$. But we have $n \geqslant 7$, hence $k \geqslant 3$. In this case we already have an NSS (Example 3.4). 
Condition (2) never fails.

Condition (3) fails iff $n-k \leq 5$. Find these cases. Recall that $k \leq(n-1) / 2$. Substitute it: $n \leq(n-1) / 2+5$. This gives $n \leq 9$. List all these pairs $(n, k)$ (with $\left.k<\frac{n}{2}\right)$.

$n=7:$ pairs $(7,2)$ and $(7,3) ; \quad n=8:$ pair $(8,3) ; \quad n=9:$ pairs $(9,2)$ and $(9,4)$.

But we already have NSSes for all these pairs. Indeed, the cases $(7,2)$ and $(8,3)$ coincide with Examples 3.1 and 3.2 respectively. The cases $(7,3)$ and $(9,4)$ are the particular cases of $n=2 k+1$ (Example 3.4). The case $(9,2)$ can be obtained from $(7,2)$ (Example 3.1) using the Step procedure.

Finally, take all the cases where the NSS is already constructed as the base of the descent. In all the other cases the descent is feasible, consequently, we have constructed an NSS for all pairs $(n, k)$ for which conditions $(1)-(3)$ hold.

Now we consider the case $\frac{k}{n} \in\left\{\frac{2}{5}, \frac{3}{5}\right\}$. Let $k=2 k_{1}, n=5 k_{1}, k_{1} \geqslant 2$. If $k_{1} \geqslant 4$, we can construct an NSS using the Step procedure and substitution $k \rightarrow n-k$ : starting with an NSS for $\left(2 k_{1}, k_{1}\right)$, we successively construct NSSes for $\left(3 k_{1}, k_{1}\right),\left(3 k_{1}, 2 k_{1}\right)$, and $\left(5 k_{1}, 2 k_{1}\right)$. If $k_{1}=2$, Example 3.7 can be applied.

If $k_{1}=3$, the pair $(n, k)=(15,6)$, and the required NSS can be obtained from Example 3.6 using the Step procedure.

\subsubsection{Case when $k \mid n$ or $(n-k) \mid n$}

Assume that $k \leq n / 2$. Then $k \mid n$, let $d=n / k$. The case $k=1$ is already considered (see 2.1 ), so here $k \geqslant 2$.

If $k \geqslant 4$, Example 3.3 shows that the NSS exists for the pair $(2 k, k)$. Using the Step procedure, we can easily rebuild this NSS into the NSS for a pair $(k d, d)$, where $d \geqslant 2$. It remains to consider cases $k=2$ and 3 .

$k=2$. It follows from the Main Theorem that $d \geqslant 4$. But we already have an NSS for the pair $(8,2)$ (Example 3.5). Using the Step procedure, we can construct NSSes for all $d>4$.

$k=3$. We already have an NSS for $(9,3)$. Using the Step procedure, we can construct an NSS for all $n$ such that $n>9$ and $3 \mid n$. We are done.

\subsection{Non-fundamental weights}

Lemma 3.10 (The Inclusion Lemma) Let $\lambda$ and $\lambda^{\prime}$ be two dominant weights such that $\lambda^{\prime} \in M(\lambda)$, and there exists an NSS in $M\left(\lambda^{\prime}\right)$. Then there exists an NSS in $M(\lambda)$.

Proof Notice that $\forall \sigma \in W \quad \sigma \lambda^{\prime} \in M(\lambda)$ and $P^{\prime}=\operatorname{conv}\left\{\sigma \lambda^{\prime}: \sigma \in W\right\} \subset P$. Then $M\left(\lambda^{\prime}\right)=\left(\lambda^{\prime}+\Phi\right) \cap P^{\prime} \subset M(\lambda)$. This means that the NSS for $\lambda^{\prime}$ is also an NSS for $\lambda$.

Remark 1 It follows from Lemma 3.10 that we already have NSSes for the major part of non-fundamental weights (a non-fundamental weight can be reduced to fundamental). However, this method does not cover all the cases, and the size of the resulting NSS grows as $\operatorname{dim} V$ does. Below, NSSes containing only 4 vectors are constructed for all such cases. 
There are two cases: all the usual coordinates of $\lambda$ are integer or all of them are non-integer. Consider these cases independently. The coordinates of vectors in the usual basis are denoted by $y_{i}$.

\subsubsection{All the coordinates of $\lambda$ are integer, $\lambda \neq(1,0, \ldots, 0,-1)$}

Definition By a shift we mean the following procedure: take a point $\lambda=\left(y_{1}, \ldots, y_{n}\right)$, fix two indices $i<j$ such that $\left|y_{i}-y_{j}\right| \geqslant 2$ and replace $\lambda$ with the point $\lambda^{\prime}$, where $\lambda^{\prime}=\left(\ldots, y_{i}-1, \ldots, y_{j}+1, \ldots\right)$ if $y_{i}>y_{j}$ and $\left(\ldots, y_{i}+1, \ldots, y_{j}-1, \ldots\right)$ otherwise.

The point $\lambda^{\prime}$ lies in $M(\lambda)$. Indeed, $M(\lambda)$ contains the point $\left(\ldots, y_{j}, \ldots, y_{i}, \ldots\right)$, the convex hull of $\lambda$ and $\left(\ldots, y_{j}, \ldots, y_{i}, \ldots\right)$ contains $\lambda^{\prime}$. Notice that after each use of the shift the value $y_{1}^{2}+\ldots+y_{n}^{2}$ diminishes by a positive integer. Indeed, let $x=$ $\max \left\{y_{i}, y_{j}\right\}, y=\min \left\{y_{i}, y_{j}\right\}$. Then $x-y \geqslant 2$, and

$$
\begin{aligned}
(x-1)^{2}+(y+1)^{2} & =x^{2}-2 x+1+y^{2}+2 y+1=x^{2}+y^{2}-2(x-y-1) \\
& \leqslant x^{2}+y^{2}-2 .
\end{aligned}
$$

This means that if we apply consequent shifts to $\lambda$, then this process cannot be infinite.

Lemma 3.11 If $n \geqslant 3$ and $\lambda$ satisfies the conditions of Subsection 3.2.1, then $M(\lambda)$ contains one of the points $(2,0, \ldots, 0,-1,-1),(1,1,0, \ldots, 0,-2)$, or $(1,1,0, \ldots, 0,-1,-1)$, and it always contains the point $(1,0, \ldots, 0,-1)$.

Proof Let $\lambda=\left(a_{1}, \ldots, a_{n}\right)$ (in the usual basis). If $\forall i a_{i} \in\{-1,0,1\}$, then, due to the fact that $\lambda \neq(1,0, \ldots, 0,-1), \lambda$ has at least 4 nonzero coordinates. Taking into account that $\sum_{1}^{n} a_{i}=0$, at least two of them are equal to 1 and two are equal to -1 . In this case, $M(\lambda)$ contains the point $(1,1,0, \ldots, 0,-1,-1)$ : split all its other coordinates into pairs $(1,-1)$ and make them zero (using the shift), then permute the remaining 4 coordinates. Applying one more shift, we yield $(1,0, \ldots, 0,-1)$.

Otherwise, if $\exists i,\left|a_{i}\right|>1$ (one of the coordinates is big), then $\max _{i, j}\left(a_{i}-a_{j}\right) \geqslant 3$. Keeping at least one coordinate big, perform the shift for the pairs of indices where $\left|a_{i}-a_{j}\right| \geqslant 2$. This process is finite. Consider a situation where we can perform no more shifts. If we still have a nonzero coordinate with the same sign as the big coordinate has, we can shift it with the coordinate of the opposite sign (their difference will obviously be $\geqslant 2$ ). Otherwise we are in the case where we have a big coordinate of one sign (without loss of generality positive) and some coordinates of the opposite sign. If the big coordinate is $\geqslant 3$, then apply a shift to this coordinate and to some negative coordinate. But we have supposed that shifts are impossible. Then the big coordinate is equal to 2 , nonzero ones among the other coordinates are either -2 or two $-1 \mathrm{~s}$. But if $M(\lambda)$ contains a point $(2,0,0, \ldots, 0,-2)$, then it also contains $(2,0, \ldots, 0,-1,-1)=\frac{1}{2}((2,0, \ldots, 0,-2)+(2,0, \ldots, 0,-2,0))$. We can easily get $(1,0, \ldots, 0,-1)$, performing one more shift.

Construct NSSes for the first three points. 
Example $3.12 \lambda=(2,0, \ldots, 0,-1,-1), n \geqslant 3$. Consider vectors

$$
\begin{gathered}
v_{1}=(1,-1,0,0, \ldots, 0), \quad v_{2}=(-1,-1,2,0, \ldots, 0), \\
v_{3}=(2,-1,-1,0, \ldots, 0), \\
v=(0,-1,1,0, \ldots, 0)=\frac{1}{2}\left(v_{1}+v_{2}\right)=v_{2}+v_{3}-v_{1} .
\end{gathered}
$$

Suppose $f=-y_{2}$, then $f\left(v_{1}\right)=f\left(v_{2}\right)=f\left(v_{3}\right)=f(v)=1$, but $v \neq v_{i}$ for any $i$. We get a contradiction.

The NSS for the point $\lambda=(1,1,0, \ldots, 0,-2), n \geq 3$ can be constructed similarly (one should multiply all the coordinates by -1 ).

Example $3.13 \lambda=(1,1,0, \ldots, 0,-1,-1) \in M(\lambda), n \geq 4$. Consider vectors

$$
\begin{gathered}
v_{1}=(1,1,-1,-1,0, \ldots, 0), \quad v_{2}=(1,-1,1,-1,0, \ldots, 0), \\
v_{3}=(0,1,0,-1,0, \ldots, 0), \quad v_{4}=(0,0,1,-1,0, \ldots, 0), \\
v=(1,0,0,-1,0, \ldots, 0)=\frac{1}{2}\left(v_{1}+v_{2}\right)=v_{1}+v_{4}-v_{3} .
\end{gathered}
$$

Suppose $f=-y_{4}$, then $f\left(v_{1}\right)=f\left(v_{2}\right)=f\left(v_{3}\right)=f\left(v_{4}\right)=f(v)=1$, but $v \neq v_{i}$ for any $i$. We get a contradiction.

Now take an arbitrary dominant weight $\lambda, n \geqslant 3$, and the corresponding set $M(\lambda)$. It follows from Lemma 3.11 and the Inclusion Lemma that an NSS for $\lambda$ exists.

It remains to consider the case $n=2, \lambda=(a,-a)$. If $|a| \geq 3$, then $\operatorname{conv}\left\{\left(\sigma\left(a_{1}\right.\right.\right.$, $\left.\left.\left.a_{2}\right)\right), \sigma \in S_{2}\right\}$ contains the points $(2,-2)$ and $(3,-3)$. But this subset is not saturated:

$$
(1,-1)=\frac{1}{2}(2,-2)=(3,-3)-(2,-2),
$$

and the vector $(1,-1)$ is not a linear combination of vectors $(2,-2)$ and $(3,-3)$ with integer positive coefficients. If, otherwise, $a \in\{0, \pm 1, \pm 2\}$, then each subset in $M(\lambda)$ is saturated (Sections 2.2 and 2.4).

\subsubsection{All the coordinates of $\lambda$ are non-integer}

Lemma 3.14 Take a point $\lambda=\left(a_{1}, a_{2}, \ldots, a_{n}\right)$ (in the usual basis), $n \geqslant 4$. If the set $\left\{a_{1}, a_{2}, \ldots, a_{n}\right\}$ contains simultaneously $\alpha+1, \alpha$, and $\alpha-1$ for some $\alpha \in \mathbb{R}$, then the set $M(\lambda)$ contains an NSS.

Proof It is easy to see that $M(\lambda)$ contains a point $v_{1}=\left(\alpha+1, \alpha, \alpha-1, a_{4}, \ldots, a_{n}\right)$, $a_{4} \neq 0$ (because $a_{4} \notin \mathbb{Z}$ ). Acting by $S_{n}$, we can get the following points from it:

$$
\begin{aligned}
& v_{2}=\left(\alpha-1, \quad \alpha, \quad \alpha+1, \quad a_{4}, \ldots, a_{n}\right), \\
& v_{3}=\left(\alpha+1, \quad \alpha-1, \quad \alpha, \quad a_{4}, \ldots, a_{n}\right), \\
& v_{4}=\left(\alpha, \quad \alpha-1, \quad \alpha+1, \quad a_{4}, \ldots, a_{n}\right) .
\end{aligned}
$$


Let us show that this set is not saturated. Indeed,

$$
\begin{gathered}
\frac{1}{2}\left(v_{1}+v_{2}\right)=\left(\alpha, \alpha, \alpha, a_{4}, \ldots, a_{n}\right), \\
v_{2}+v_{3}-v_{4}=\left(\alpha, \alpha, \alpha, a_{4}, \ldots, a_{n}\right) .
\end{gathered}
$$

If $f=\frac{y_{4}}{a_{4}}$, then $f\left(v_{1}\right)=f\left(v_{2}\right)=f\left(v_{3}\right)=f\left(v_{4}\right)=f(v)=1$. But $v \neq v_{i}$ for any $i$, this means that $v$ is not a $\mathbb{Z}_{+}$-combination of $v_{i}$.

Lemma 3.15 (The Good Triple Lemma) Let $\lambda=\left(a_{1}, \ldots, a_{n}\right), n \geqslant 4$, and all $a_{i}$ be non-integer. If the collection $a_{1}, \ldots, a_{n}$ contains at least three different values, then $M(\lambda)$ contains a point of form $\left(\alpha+1, \alpha, \alpha-1, a_{4}, \ldots, a_{n}\right)$.

Proof Perform several shifts preserving the condition that the set $\left\{a_{1}, \ldots, a_{n}\right\}$ contains at least 3 different elements. Suppose further shifts are impossible (we mentioned above that, starting from any position, only a finite number of shifts is possible). Consider $a_{\max }=\max \left\{a_{1}, \ldots, a_{n}\right\}, a_{\min }=\min \left\{a_{1}, \ldots, a_{n}\right\}, a_{\text {mid }} \in\left\{a_{1}, \ldots, a_{n}\right\}$, $a_{\text {mid }} \neq a_{\text {max }}, a_{\text {mid }} \neq a_{\text {min. }}$. If $a_{\text {max }}-a_{\text {mid }} \geqslant 3$, then we can apply the shift to $a_{\max }$ and $a_{\text {mid }}$, thus we obtain three different values of coordinates $a_{\text {min }}, a_{\text {mid }}+1, a_{\text {max }}-1$. Similarly, if $a_{\text {mid }}-a_{\min } \geqslant 3$, then at least one more shift is possible. So we yield $a_{\text {max }}-a_{\text {mid }}, a_{\text {mid }}-a_{\text {min }} \in\{1,2\}$. If $a_{\text {max }}-a_{\text {mid }}=a_{\text {mid }}-a_{\text {min }}=1$, we have already found a point of necessary type in $M(\lambda)$. Up to symmetry, one of the two cases is possible: either $a_{\mathrm{min}}=a_{\mathrm{mid}}-2, a_{\mathrm{max}}=a_{\mathrm{mid}}+2$, or $a_{\mathrm{min}}=a_{\mathrm{mid}}-1, a_{\mathrm{max}}=a_{\mathrm{mid}}+2$. Consider these two cases.

In the first case, apply the shift to $a_{\max }$ and $a_{\min }$. This operation gives us the required triple $\left(a_{\max }-1, a_{\mathrm{mid}}, a_{\min }+1\right)$.

In the second case, $a_{\text {min }}=a_{\text {mid }}-1, a_{\text {max }}=a_{\text {mid }}+2$, and we know that $\lambda$ has at least 4 coordinates. If there are 4 different values among them, the fourth will inevitably form a triple of form $(\alpha+1, \alpha, \alpha-1)$ with two of $a_{\mathrm{max}}, a_{\mathrm{mid}}, a_{\mathrm{min}}$. Otherwise $a_{i} \in\left\{a_{\max }, a_{\text {mid }}, a_{\min }\right\}$ for any $i$. But $n \geqslant 4$, this means that at least one of the values $\left(a_{1}, a_{2}, \ldots, a_{n}\right)$ occurs twice. Suppose $n=4$ (we need only $4 a_{i}$ 's). The multiplicities of $\left(a_{\mathrm{max}}, a_{\mathrm{mid}}, a_{\mathrm{min}}\right)$ may be as follows: $(\widehat{1,1,2}),(\widehat{1,2}, 1),(\widehat{2,1,1})$. Apply the shift to the coordinates marked with the hat. We get one of the following collections: $\left(a_{\text {mid }}+1, a_{\text {mid }}, a_{\text {mid }}, a_{\text {mid }}-1\right),\left(a_{\text {mid }}+1, a_{\text {mid }}+1, a_{\text {mid }}, a_{\text {mid }}-1\right),\left(a_{\text {mid }}+2, a_{\text {mid }}+\right.$ $\left.1, a_{\text {mid }}, a_{\text {mid }}\right)$. Each of them contains a triple of form $(\alpha+1, \alpha, \alpha-1)$. But this means that here we also find a triple of form $(\alpha+1, \alpha, \alpha-1)$.

Lemma 3.16 Let $\lambda=\left(a_{1}, \ldots, a_{n}\right)$ be a dominant weight, $n \geqslant 4, \exists i$ with $\left|a_{i}\right|>1$, and all $a_{i} \notin \mathbb{Z}$. If $M(\lambda)$ does not contain a point of the form $\left(\alpha+1, \alpha, \alpha-1, a_{4}, \ldots, a_{n}\right)$, then

$$
\lambda=\left(\frac{2 n-2}{n},-\frac{2}{n},-\frac{2}{n}, \ldots,-\frac{2}{n}\right) \text { or } \lambda=\left(\frac{2}{n}, \frac{2}{n}, \ldots, \frac{2}{n},-\frac{2 n-2}{n}\right) .
$$

Proof If the collection $a_{1}, \ldots, a_{n}$ contains at least 3 different elements, then we can use the Good Triple Lemma and show that $M(\lambda)$ contains a point of the desired form. 
This means that $\forall i a_{i} \in\left\{a_{\max }, a_{\min }\right\}$. Without loss of generality, we may suppose that $a_{\max }>1$, and $a_{\min }<0$ (otherwise multiply all $a_{i}$ by -1 ).

If $a_{\min }<-1$, then apply the shift to $a_{\min }$ and $a_{\max }$. Thus we get $a_{\min }+1$ and $a_{\max }-1$ among the values of the coordinates, and still at least one of $a_{\min }$ and $a_{\max }$ is presented (since $n \geqslant 4>3$ ). Using the Good Triple Lemma, we get a contradiction.

We see that $-1<a_{\min }<0$. If the collection $\left(a_{1}, \ldots, a_{n}\right)$ contains $a_{\max }$ at least for 2 times, then apply the shift to $a_{\min }$ and $a_{\max }$. Now we have $a_{\max }, a_{\min }+1>0$ and at least one time $a_{\mathrm{min}}$ among the values of coordinates: all the coordinates cannot be positive. This gives us a contradiction with the Good Triple Lemma.

Then $a_{\max }$ enters only once in $\left(a_{1}, \ldots, a_{n}\right)$. If $a_{\max }>2$, apply the shift to $a_{\min }$ and $a_{\max }$. We get that $a_{\max }-1>1, a_{\min }+1<1$ and $a_{\min }$ are among the values of coordinates, which gives us a contradiction with the Good Triple Lemma.

We see that the collection has a form $\left(a_{\max }, a_{\min }, a_{\min }, \ldots, a_{\min }\right), 1<a_{\max }<2$, $-1<a_{\min }<0$. Let $a_{\min }=-\frac{k}{n}$. We have $(n-1) a_{\min }+a_{\max }=0$ from the initial conditions. This yields $a_{\max }=\frac{k(n-1)}{n}$. But $a_{\max }<2$. Consequently,

$$
\frac{k(n-1)}{n}<2 \Rightarrow k(n-1)<2 n \Rightarrow k<\frac{2 n}{n-1}<3,
$$

because $n \geqslant 4$. Taking into account that $a_{\max }>1$, we get $k=2, a_{\max }=\frac{2 n-2}{n}$, $a_{\min }=-\frac{2}{n}$. But in the beginning of the case we could change the signs at all the coordinates. Thus, we have two cases: $\lambda=\left(\frac{2 n-2}{n},-\frac{2}{n},-\frac{2}{n}, \ldots,-\frac{2}{n}\right)$ and $\lambda=$ $\left(-\frac{2 n-2}{n}, \frac{2}{n}, \frac{2}{n}, \ldots, \frac{2}{n}\right)$.

Applying the Lemmas, we see that in the case, when $a_{i} \notin \mathbb{Z}, \exists i,\left|a_{i}\right|>1, n \geqslant 4$, we have not constructed an NSS only in these two cases. In all the other cases the NSS exists due to Lemma 3.14. Let us construct an NSS in these two cases. We may assume that $\lambda=\left(\frac{2 n-2}{n},-\frac{2}{n},-\frac{2}{n}, \ldots,-\frac{2}{n}\right)=2 e_{1}$. Let

$$
v_{1}=2 e_{1}, \quad v_{2}=2 e_{2}, \quad w=2 e_{3}, \quad v_{3}=e_{1}+e_{3} \in M(\lambda), \quad v_{4}=e_{2}+e_{3} \in M(\lambda) .
$$

Then $v_{1}, v_{2}, v_{3}, v_{4}$ form an NSS. Indeed, we have

$$
v=e_{1}+e_{2}=\frac{1}{2}\left(v_{1}+v_{2}\right)=v_{1}+v_{4}-v_{3}, \quad f=x_{1}+x_{2}+x_{3}-3 x_{n} .
$$

Then $f\left(v_{1}\right)=f\left(v_{2}\right)=f\left(v_{3}\right)=f\left(v_{4}\right)=f(v)=2$, and $v \neq v_{i}$ for any $i$. But 2 cannot be represented as a sum of more than one $2 \mathrm{~s}$. We get a contradiction.

Now it remains to consider the cases $n=3$ and $n=2$.

In the case $n=3$ we suppose that the fractional parts of all coordinates are equal to $\frac{2}{3}$ (otherwise change $\lambda$ for $-\lambda$, as we have done earlier). If $\lambda=\left(\frac{2}{3}, \frac{2}{3},-\frac{4}{3}\right)$, then $M(\lambda)$ is hereditary normal (see 2.5). Below we construct an NSS for $\lambda=$ $\left(\frac{5}{3},-\frac{1}{3},-\frac{4}{3}\right)=3 e_{1}+e_{2}$, then, using the Inclusion Lemma, show the existence of an NSS for all other points $\lambda$. Let

$$
v_{1}=e_{1}=\frac{2}{3}\left(3 e_{1}+e_{2}\right)+\frac{1}{3}\left(e_{2}+3 e_{3}\right),
$$




$$
\begin{gathered}
v_{2}=2 e_{1}+2 e_{2}=\frac{1}{2}\left(3 e_{1}+e_{2}\right)+\frac{1}{2}\left(e_{1}+3 e_{2}\right), \\
v_{3}=3 e_{1}+e_{2}, \quad v=2 e_{1}+e_{2}=v_{1}+\frac{1}{2} v_{2}=v_{3}-v_{1} .
\end{gathered}
$$

If $f=x_{1}-x_{3}$, then $f\left(v_{1}\right)=1, f\left(v_{2}\right)=2, f\left(v_{3}\right)=3$, and $f(v)=2$. But $v$ is equal neither to $v_{2}$, nor to $2 v_{1}$. We get a contradiction.

Lemma 3.17 Suppose that $\lambda=\left(a_{1}, a_{2}, a_{3}\right)$ (in the usual basis) is a dominant weight such that the fractional parts of all $a_{i}$ are equal to $\frac{2}{3}$. Suppose also that there exists an index $i$ with $\left|a_{i}\right|>1$, and $\lambda \neq\left(\frac{2}{3}, \frac{2}{3},-\frac{4}{3}\right)$. Then $M(\lambda)$ contains a point $\left(\frac{5}{3},-\frac{4}{3},-\frac{1}{3}\right)$.

Proof It follows from the conditions of the Lemma that $\exists i, a_{i} \geq \frac{5}{3}$. Indeed, otherwise we have at least 2 positive coordinates, each of them $\leq \frac{2}{3}$, but due to the condition of the Lemma there exists an $a_{i}$ such that $\left|a_{i}\right|>1$. Suppose it is $a_{1}$. We have $a_{1}=$ $-a_{2}-a_{3} \geqslant-\frac{4}{3}$. This means that $\lambda=\left(-\frac{4}{3}, \frac{2}{3}, \frac{2}{3}\right)$. We get a contradiction.

If only one coordinate of $\lambda$ is positive, and it is equal to $\frac{5}{3}$, then $\lambda=\left(\frac{5}{3},-\frac{4}{3},-\frac{1}{3}\right)$, and the Lemma is proved. Otherwise either $\lambda$ has two positive coordinates, or one of them is $\geqslant \frac{8}{3}$. In both cases we can apply the shift to a positive and a negative coordinate, such that after it $\lambda$ still has a coordinate $\geq \frac{5}{3}$, and so on.

Consider the case $n=2$. Suppose that $\lambda=\left(\frac{a}{2},-\frac{a}{2}\right), a \in \mathbb{N}, a$ is odd. Then for $a=3$ it was already checked that $M(\lambda)$ is hereditary normal (see 2.3 ). If $a \geqslant 5$, let us construct an NSS. Set

$$
v_{1}=\left(\frac{3}{2},-\frac{3}{2}\right), v_{2}=\left(\frac{5}{2},-\frac{5}{2}\right) .
$$

Then $\left(\frac{1}{2},-\frac{1}{2}\right)=\frac{1}{3} v_{1}=2 v_{1}-v_{2} \notin \mathbb{Z}_{+}\left(v_{1}, v_{2}\right)$.

So we have constructed non-saturated subsets in the sets of weights for all the representations not listed in the Main Theorem.

Acknowledgements The author is grateful to her scientific supervisor I.V. Arzhantsev for the formulation of the problem and fruitful discussions. Thanks are also due to I.I. Bogdanov for useful comments. Special thanks go to the referees whose suggestions helped to shorten several proofs and improve the presentation.

\section{References}

1. Arzhantsev, I.V.: On the normality of the closures of spherical orbits. Func. Anal. Prilozh. 31(4), 66-69 (1997) (in Russian), English transl.: Func. Anal. Appl. 31, No. 4 (1997), 278-280

2. Bobiński, G., Zwara, G.: Normality of orbit closures for directing modules over tame algebras. J. Algebra 298, 120-133 (2006)

3. Carrell, J.B., Kurth, A.: Normality of torus orbit closures in G/P. J. Algebra 233, 122-134 (2000) 
4. Chindris, C.: Orbit semigroups and the representation type of quivers. arXiv:0708.3413v1

5. Dabrowski, R.: On normality of the closure of a generic torus orbit in $G / P$. Pac. J. Math. 192(2), 321-330 (1996)

6. Fulton, W.: Introduction to Toric Varieties. Princeton University Press, Princeton (1993)

7. Harary, F.: Graph Theory. Addison-Wesley, Reading (1969)

8. Humphreys, J.E.: Introduction to Lie Algebras and Representation Theory. GTM, vol. 9. Springer, Berlin (1978)

9. Kemph, G., Knudsen, F., Mumford, D., Saint-Donat, B.: Toroidal Embeddings I. LNM, vol. 339. Springer, Berlin (1973)

10. Klyachko, A.A.: Toric varieties and flag varieties. Tr. Mat. Inst. Steklova 208, 139-162 (1995) (in Russian), English transl.: Proc. Steklov Inst. Math. 208 (1995), 124-145

11. Morand, J.: Closures of torus orbits in adjoint representations of semisimple groups. C.R. Acad. Sci. Paris Sér. I Math. 328(3), 197-202 (1999)

12. Ohsugi, H., Hibi, T.: Normal polytopes arising for finite graphs. J. Algebra 207, 409-426 (1998)

13. Simis, A., Vasconcelos, W., Villarreal, R.: The integral closure of subrings associated to graphs. J. Algebra 199, 281-299 (1998)

14. Sturmfels, B.: Gröbner Bases and Convex Polytopes. University Lecture Series, vol. 8. AMS, Providence (1996)

15. Sturmfels, B.: Equations defining toric varieties. Proc. Sympos. Pure Math. 62, 437-449 (1997)

16. Vinberg, E.B., Onishchik, A.L.: Seminar on Lie Groups and Algebraic Groups. Nauka, Moskva (1988) (in Russian). English transl.: Lie Groups and Algebraic Groups, Berlin, Springer 1990

17. White, N.: The basis monomial ring of a matroid. Adv. Math. 24, 292-297 (1977) 